\title{
Nicotine Response to Interval Training in Male Smokers
}

\author{
MAHA M. AZZAM, M.Sc.*; SAMAH M. ISMAIL, Ph.D.**; ZAHRAA M. HASSAN, Ph.D.** and \\ MAHA H. SABRY, M.D.*** \\ The Department of Physical Therapy for Cardiovascular/Respiratory Disorder and Geriatrics, New Cairo Hospital*, \\ Faculty of Physical Therapy, Cairo University** and The Depatment of Copmlementary Medicine, National Research Center***
}

\begin{abstract}
Background: Tobacco overtaking, sedentary lifestyle and low fitness considered as the main risk factors for diseases that leads to death, such as, coronary heart disease, stroke, obesity and hypertension which are the most common causes of morbidity and mortality.

Aim of Study: The purpose of this study was to find out the effect of interval training on nicotine in male smokers.

Subject and Methods: The study was conducted on thirty smokers in New Cairo Hospital started at August 2017 and had been finished at December 2018, their age ranged from 20 to 40 years their BMI ranged from 25 to $29.9 \mathrm{~kg} / \mathrm{m}^{2}$ all subjects were thoroughly evaluated before and after the exercise protocol application. Initial assessment were included measurements of body weight, body height, and nicotine blood levels, all were free from musculoskeletal disorders or cardiovascular disorders, then subjects started treatment program as following, performing a supervised running on treadmill in pattern of high intensity interval training for 30 minutes, 3 sessions per week for 8 weeks. Each training session was consisted of 5 minutes of warming-up walking, 10 minutes of high intensity interval training consist of 2 repeats of ( $1 \mathrm{~min}$ running at $85-95 \%$ of MHR then followed by $4 \mathrm{~min}$ recovery at $60-70 \%$ of MHR), and ended with 5 minutes of cool-down walking.
\end{abstract}

Results: Statistical analysis showed a significant improvement (decrease) in cotinine level (nicotine) by $39.94 \%$.

Conclusion: It is recommended for smokers to participate in high intensity interval training to decrease smoking craving, and then smoking cessation.

Key Words: Nicotine in blood - Treadmill - High intensity interval training.

\section{Introduction}

CIGARETTES are the most common source of nicotine, smoked tobacco contains additional harmful constituents and chemicals, which have detrimental effects on the respiratory system [1] with

Correspondence to: Dr. Maha M. Azzam, The Department of Physical Therapy for Cardiovascular/Respiratory Disorder and Geriatrics, New Cairo Hospital
Egypt's smoking population steadily increasing, cigarettes and smoking tobacco will continue to grow over the following years. Both sexes and all income segments of the population are now smoking, making this an even more widely accepted habit [2]. Given the numerous diseases caused by tobacco use, the health care cost of treating these diseases is substantial. In 2005, estimates indicated that about 3.4 billion EGP (US\$ 616 million) were spent annually in Egypt to treat the diseases caused by tobacco use [3] Nicotine's addictive nature includes psychoactive effects, drug-reinforced behavior, compulsive use, relapse after abstinence, physical dependence and tolerance [4] . Studies have proven that smoking cigarettes causes cancers of the bladder, oral cavity, pharynx, larynx (voice box), esophagus, cervix, kidney, lung, pancreas, and stomach, and causes acute myeloid leukemia. It also causes heart disease and stroke [5]. Nicotine can be quantified in blood, plasma, or urine to confirm a diagnosis of poisoning or to facilitate a forensic autopsy. Urinary or salivary cotinine concentrations are frequently measured. unfortunately passive exposure to cigarette smoke can result in significant accumulation of nicotine, followed by the appearance of its metabolites in various body fluids [7], interval training program is an effective adjunct no pharmacological management of hypertension and a means of up regulation of (HDL) [8] . HIIT, high intensity interval training, (combined with a smoking quit program) will result in higher quit rates than a more moderate physical activity intervention [9]. The results of two studies which examined the acute and chronic effects of vigorous exercise on nicotine withdrawal symptoms in a group of sedentary women, during a 12 week cognitive behavioral smoking cessation program. In addition to receiving smoking cessation support, individuals were assigned to either an exercise program or to a contact control session 
three times per week. Immediate reductions in nicotine withdrawal and cigarette cravings were observed following 30-40 minutes of high intensity exercise relative to the controls [6]. Some people who smoke but exercise assume that strengthening their lungs by working out must cancel the negative effects of smoking, they found that those who smoked, but who participated in vigorous physical activity at least twice a week, were nearly 30 percent less likely to get lung cancer than those women who were inactive [10]. A number of studies suggested that vigorous exercise $(60-85 \%$ of heart rate reserve) can be a useful aid to quit smoking [6]. High interval training is defined as repetitions of high intensity work followed by periods of rest or low activity. Interval training works both the aerobic and the anaerobic systems. During the high intensity effort, the anaerobic system uses the energy stored in the muscles for short bursts of activity [11]

\section{Subjects and Methods}

\section{I- Subject:}

Thirty men smokers, their Body Mass Index (BMI) ranged from $25-29.9 \mathrm{~kg} / \mathrm{m}^{2}$ and they were sedentary (not participating in regular aerobic exercise), are involved in this study. Subjects are clinically diagnosed. They were selected from New Cairo Hospital. Their ages range from 20 to 40 years old. The study was conducted at smoking cessation clinic at New Cairo Hospital, started on August 2017 and completed December 2018. Exercise program was running using Electrical Treadmill. The patients perform exercise at high intensity (10 minutes of high intensity interval training consist of 2 repeats of ( $1 \mathrm{~min}$ running at $85-95 \%$ of MHR then followed by $4 \mathrm{~min}$ recovery at 60 $70 \%$ of MHR)), 3 sessions per week for 8 weeks without any smoking cessation medications.

- All subjects were evaluated before and after treatment protocol application.

\section{Inclusion criteria:}

All patients were:

- Men.

- Smokers "cigarette only".

- Age range from 20-40 years old.

- BMI ranged from 25-29.9.

\section{Exclusion criteria:}

All patients had one of the following were excluded from study:

- Hypertensive patient.

- Smoking any other smoking types.
- Taking medication for any disease.

- Any liver diseases.

- Alcohol intake.

- History of chronic chest disease.

- History of cardiovascular disorder.

-With musculoskeletal disorder like tendon strain, muscle strain or spasm.

\section{II- Instrumentation:}

A- Evaluation tools and equipment:

1-Laboratory investigation (Nicotine level): $3 \mathrm{ml}$ of blood were taken from each subject before 1 st session. To be analyzed by Stat Fax-2100 US to measure nicotine level in blood. (Applied in Biochemistry Department Lab in El-Kasr ElEney.

Nicotine level was measured before and after treatment protocol application.

2- Height and weight scale (floor type model ZT120, Made in China): The measured height and weight was used to calculate Body Mass Index (BMI) of each patient to fulfill the inclusion criteria of the study.

3- Mercury sphygmomanometer (Diplomat presameter, made in Germany) and stethoscope (Rooster duplex, made in Germany): It was used to measure the blood pressure before and after each exercise training session to make sure it's stable.

4- Pulsometer JAX 207 finger pulse oximeter, made in China: Used to detect the pulse rate before and after exercise, and to control the exercise intensity within the pre calculated training heart rate during every session.

\section{B- Training equipment:}

JS-1688 ELECTRICAL TREADMILL Model (JS 1688, made in China).

\section{III- Procedures:}

The study protocol was explained in details for every subject before the initial assessment. A complete history and physical examination were taken for all smokers with particular attention paid to identifying long-term complications of smoking. A written informed consent was signed by each patient before participation in the study as an agreement to be included in the present study.

\section{Results}

This study was reviewed and was approved by the Ethics Committee of Faculty of Physical Therapy, Cairo University. 


\section{A- Evaluation procedure:}

1-Lab investigation: All subjects were subjected to all the following protocol: $3 \mathrm{ml}$ of blood were taken from each subject before 1 st session to measure nicotine level in blood.

2-Body mass index evaluation: A height and weight scale was used to measure height and weight to calculate body mass index: $\mathrm{BMI}=$ body weight in kilograms/height in meter squared. BMI= (Weight) $/(\text { Height })^{2}$ [18]

3- Maximum heart rate measure: The exercise intensity has been prescribed as training heart rate depending on each subject's maximum heart rate (HR Max) and resting heart rate (HR rest), MHR $=220$-AGE, training heart rate was applied $85-95 \%$ of MHR during high intensity phase and $60-70 \%$ of MHR during recovery phase.

4- Nicotine levels in blood was measured at the beginning and end of the study ( 8 weeks) for all subjects (Fig. 1).

\section{B- Training procedure:}

All patients were individually instructed at the first training session about high intensity interval training and how to stop it when they cannot continue. All instructions were given to the patients to be familiar with high intensity interval training. All of the 30 patients in the study group were performing a supervised running on treadmill in pattern of high intensity interval training for 30 minutes, 3 sessions per week for 8 weeks. Each training session was consisted of 5 minutes of warming-up activities consisting of walking, 20 minutes of high intensity interval training consist of 2 repeats of ( $1 \mathrm{~min}$ running at $85-95 \%$ of MHR then followed by $4 \mathrm{~min}$ running at $60-70 \%$ of MHR, and ended with 5 minutes of cool-down activities consisting of walking.

\section{Statistical analysis:}

The data obtained from study group were statistically analyzed to compare the difference within study group for pre and post measures. The statistical analysis was conducted by using statistical SPSS Package program Version 25 for Windows (SPSS, Inc., Chicago, IL). Data was normally distributed by using Shapiro-Wilk test (parametric data). Additionally, testing for the homogeneity of variance revealed that there was no significant difference $(p>0.05)$. Descriptive statistics included the mean and standard deviation for all variables. Paired $t$-test to compare between pre and posttreatment within study group for cotinine variables. All statistical analyses were significant at level of probability $\left(p^{<} \_0.05\right)$.
Statistical analysis showed a significant improvement (decrease) in cotinine by $39.94 \%$ in the pre measure to post measure within the study group.

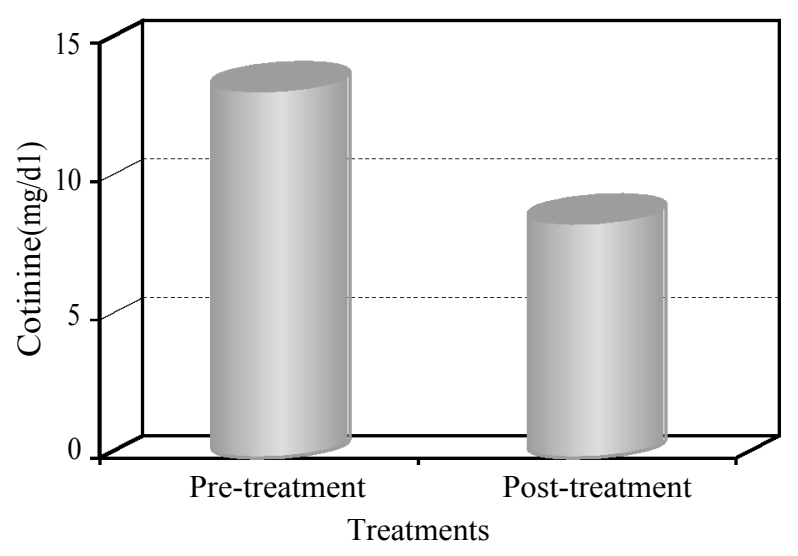

Fig. (1)

\section{Discussion}

The purpose of this study was to evaluate the effect of high intensity interval training on smokers, data obtained from study group before and after the study.

High intensity interval training seems to be a better approach for the prevention and management of the adolescent obesity and the metabolic, cardiovascular, and hormonal disorders observed in this population than does moderate intensity interval training. Therefore, those findings provide an important first step toward an evidence basis for the utilization of high intensity interval training as an exercise strategy for the young obese women [12] .

Functional Magnetic Resonance Imaging (FMRI) was used to investigate how the brain processes images of cigarettes after performing exercise, showing shifts in regional brain activation in response to smoking-related images, following exercise. It had shown that changing of emotional processing for smoking-related stimuli is linked to the pathophysiology of cigarettes craving [13]

After no exercise the smokers showed heightened activity in response to the images in areas of the brain associated with reward-processing and visual attention. While after exercise the same areas of activation were not observed, this reflected a kind of "default mode" in the brain. The smokers also reported lower cravings for cigarettes after exercise compared with when they had been inactive, researchers suggested that completing the exercise may raise the mood (possibly through increases in dopamine) which reduces the salience or importance of wanting a cigarette. Another 
possibility is that exercise may cause shift in blood flow to areas of the brain less involved in anticipation of reward and pleasure generated by smoking images [14].

High intensity interval training, (combined with a quit program) will result in higher quit rates than a more moderate physical activity intervention [9]

A number of studies to examine this issue and they suggested that vigorous exercise $(60-85 \%$ of heart rate reserve) can be a useful aid to quit smoking. To the best of our knowledge, there is a lack of empirical evidence to document if there are beneficial effects on the physiological symptoms of people who continue smoking [6]

Completing the exercise may raise the mood (by increasing dopamine) which reduce craving to smoking cigarette. Another possibility is that exercise may cause a shift in blood flow to areas of the brain less involved of anticipation of reward and pleasure generated by smoking images [16]

A short bout of moderate intensity exercise did not alter desire to smoke among abstaining adolescent smokers and may negatively impact affective responses during exercise [15]. Both moderate and vigorous intensity exercise were beneficial in alleviating the desire to smoke only during and 5 min after exercise, but only moderate intensity exercise appeared to reduce withdrawal symptoms and improve mood up to $5 \mathrm{~min}$ after exercise. No effects lasted for 30min. Vigorous exercise was associated with adverse mood outcomes during exercise, but not afterwards [16].

The mechanism of beneficial effect of exercise on withdrawal and cravings is not clear enough. But exercise has been shown to have some similarities to smoking in it's effects on stimulating the central nervous system and neurobiological processes in the brain [17]

\section{Conclusion:}

Based on the findings of this study, it could be concluded that there is improvement (decrease) in the cotinine after high intensity interval training in smokers.

\section{References}

1- AMANN M.: Pulmonary system limitations to endurance exercise performance in humans. Exp. Physiol., 97: 3118, 2012.

2- HAJJAR R.R., ATLI T., AL-MANDHARI Z., OUDRHIRI M., BALDUCCI L. and SILBERMANN M.: Prevalence of aging population in the Middle East and its implications on cancer incidence and care. Annals of Oncology, 24 (Suppl-7), vii11-vii24, 2013.
3- FOUAD H., AWA F.E., NAGA R.A.E., EMAM A.H., LABIB S., PALIPUDI K.M. and TALLEY B.: Prevalence of tobacco use among adults in Egypt, 2009. Global health promotion, 23 (2-Suppl), 38-47, 2012.

4- HOLBROOK and BRADLEY D.: "The effects of nicotine on human fetal development". Birth Defects Research Part C: Embryo Today: Reviews, 108 (2): 181-192, 2016.

5- World Health Organization: WHO study group on tobacco product regulation: Report on the scientific basis of tobacco product regulation: Fifth report of a WHO study group, (Vol. 989). World Health Organization, 2015.

6- MARCUS B.H., BOCK B., LEWIS B.A., HOGAN J., KING T.K., ALBRECHT A.E. and ABRAMS D.B.: The efficacy of moderate-intensity exercise as an aid for smoking cessation in women: A randomized controlled trial. Nicotine \& Tobacco Research, 7 (6): 871-80, 2016.

7- BASELT and RANDALL CLINT: Disposition of Toxic Drugs and Chemicals in Man (10 th ed.). Biomedical Publications, pp. 1452-6. ISBN 978-0-9626523-9-4, 2014.

8- LAMINA S. and OKOYE G.C.: Therapeutic effect of a moderate intensity interval training program on the lipid profile in men with hypertension: A randomized controlled trial. Niger. J. Clin. Pract., 15: 42-7, 2012.

9- PAVEY T.G., GARTNER C.E., COOMBES J.S. and BROWN W.J.: Assessing the effectiveness of High Intensity Interval Training (HIIT) for smoking cessation in women: HIIT to quit study protocol. BMC Public Health, 15 (1), 1309, 2015

10- THOMPSON T.P., TAYLOR A.H., GREAVES C.J., TAY LOR R.S., GREEN C., WARREN F.C. and CAMPBELL J.L.: A pilot randomised trial to assess the methods and procedures for evaluating the clinical effectiveness and cost-effectiveness of Exercise Assisted Reduction then Stop (EARS) among disadvantaged smokers. Health Technology Assessment (Winchester, England), 18 (4): $1,2014$.

11-GIBALA M.J. and GILLEN J.B.: Is high-intensity interval training a time-efficient exercise strategy to improve health and fitness? Applied Physiology, Nutrition, and Metabolism, 39 (3): 409-12, 2013.

12- RACIL G., BEN OUNIS O., HAMMOUDA O. and KALLEL A.: Effects of high vs. moderate exercise intensity during interval training on lipids and adiponectin levels in obese young females H. Zouhal, K. Chamari, M. Amri Received: 8 April 2013/Accepted: 21 June 2013/Published online: 4 July 2013-Springer-Verlag Berlin Heidelberg 2013.

13- HAASOVA M., WARREN F.C., USSHER M., VAN RENSBURG K.J., FAULKNER G., CROPLEY M. and TAYLOR A.H.: The acute effects of physical activity on cigarette cravings: Exploration of potential moderators, mediators and physical activity attributes using individual participant data (IPD) meta-analyses. Psychopharmacology, 231 (7): 1267-75, 2014.

14- SCERBO F., FAULKNER G., TAYLOR A. and THOMAS S.: Effects of exercise on cravings to smoke: The role of exercise intensity and cortisol. Journal of Sports Sciences, 28 (1): 11-9, 2010.

15- EVERSON E.S., DALEY A.J. and USSHER M.: Does exercise have an acute effect on desire to smoke, mood 
and withdrawal symptoms in abstaining adolescent smokers? Addictive Behaviors, 31 (9): 1547-58, 2006.

16- EVERSON E.S., DALEY A.J. and USSHER M.: The effects of moderate and vigorous exercise on desire to smoke, withdrawal symptoms and mood in abstaining young adult smokers. Mental Health and Physical Activity, 1 (1): 26-31, 2008.

17- ROBERTS V., MADDISON R., SIMPSON C., BULLEN
C. and PRAPAVESSIS H.: The acute effects of exercise on cigarette cravings, withdrawal symptoms, affect, and smoking behaviour: Systematic review update and metaanalysis. Psychopharmacology, 222 (1): 1-15, 2012.

18- SEDEAUD A., MARC A., MARCK A., DOR F., SCHIPMAN J., DORSEY M. and TOUSSAINT J.F.: BMI, a performance parameter for speed improvement. PloS one, 9 (2): e90183, 2014

\section{تطبيق التمارين المتقطعة عالية الشدة على المدخنين لتقليل نسبة النيكوتين فى الدمام}

$$
\text { الهدف: لمعرفة فاعلية التمارين المتقطعة عالية الشدة على نسبة النيكوتين في دم المدخنين. }
$$

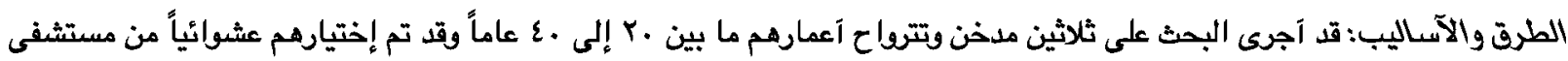

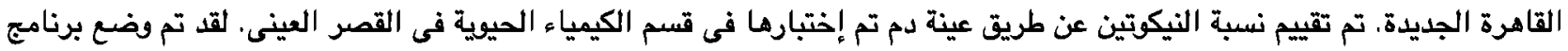
العلاج لمدة ثمانية آسابيع. النتائج:وقد آسفرت النتائج عن وجود دلالة إحصائية لتآثير التمارين المتقطعة عالية الشدة فى تقليل نسبة النيكوتين فى دم المدخنين. الخلاصة: من هذه النتائج يمكن الإستدلال على آن التمارين المتقطعة عالية الشدة لها تآثير مباشر على تقليل نسبة النيكوتين فى دم 